Published in final edited form as:

Urol Pract. 2018 July ; 5(4): 279-285. doi:10.1016/j.urpr.2017.05.002.

\title{
Patient Portal Usage in Pediatric Urology: Is it Meaningful Use for Everyone?
}

\section{Ruth A. Bush, PhD, MPH,}

Hahn School of Nursing and Health Science, Beyster Institute for Nursing Research, University of San Diego, San Diego, California

Clinical Research Informatics, Rady Children's Hospital San Diego, San Diego, California

\section{Andrew C. Richardson, MS,}

Clinical Research Informatics, Rady Children's Hospital San Diego, San Diego, California

Diana Cardona-Grau, MD,

Department of Urology, University of California San Diego, San Diego, California

\section{Hena Din, MPH,}

Department of Surgery, Rady Children's Hospital, San Diego, California

Cynthia L. Kuelbs, MD, and

Department of Pediatrics, University of California San Diego, San Diego, California

\section{George J. Chiang, MD}

Department of Urology, University of California San Diego, San Diego, California

Rady Children's Institute of Genomic Medicine, Rady Children's Hospital San Diego, San Diego, California

\section{Abstract}

Introduction-The Affordable Care Act promotes multiple directives for meaningful use of the Electronic Health Record, such as patient/provider portals, to increase patient engagement. Although portal use is common within adult healthcare, little information exists regarding pediatric portal use. We examined pediatric urology patient portal enrollment and activation patterns at a tertiary pediatric hospital in Southern California by race/ethnicity, preferred language, gender, and residential region.

Methods-Retrospective Electronic Health Record analysis of enrollment in patient portal from January 2010 to May 2016 among 10,464 patients with at least one outpatient urology clinic visit. Differences in adoption rates were examined using logistic regression for the following categories: activated (or caregiver activated); code accepted not activated; declined; or activated/then deactivated.

Results-Overall, $46.5 \%$ of patients/caregivers activated the portal. Primarily Spanish-speaking patients were less likely to activate (OR $0.25, p<.001)$ than English-speaking patients. Males (OR $0.89, p=.004$ ); those self-identifying racially as Other (not White, Asian, or African American) (OR 0.47, $p<.001$ ); and Hispanic patients (OR 0.49, $p<.001$ ) were less likely to activate. Suburban patients were up to 3 times more likely to activate portals than central urban patients depending on the region (OR 2.94, $p<.001)$. Multivariate logistic regression demonstrated 
Spanish-speaking patients were 3 times less likely to activate while controlling for demographic and region variables.

Conclusions-Primary language and socioeconomic factors may be significant barriers to portal adoption. Patient education to reduce these barriers may increase portal acceptance and increase meaningfulness to the portal for patients/parents and providers.

\section{Keywords}

electronic health record; health information technology; meaningful use; patient engagement; patient portal

\section{Introduction}

The patient portal is, "a secure online website that gives patients convenient 24-hour access to personal health information." ${ }^{1}$ Patient portals (referred here as portals) allow patients to send secure messages to clinical staff, view patient-specific educational materials, schedule appointments, request prescription refills, and manage bills. ${ }^{2}$ As part of the Affordable Care Act, multiple directives for meaningful use of the Electronic Health Record (EHR) have been promoted including the increased use and adoption of a portal with the goal of optimizing efficiency of care. ${ }^{3}$ Although portals are increasingly common, there is little information regarding their use as an engagement tool connecting patients, caregivers, and healthcare teams as partners. ${ }^{4-7}$

Adult patients who identify as racial and ethnic minorities tend to underutilize portals for a variety of reasons including: a lack of interest in the technology; not feeling the portal was helpful; physicians not discussing the portal with them; or lack of a computer or internet access. ${ }^{8}$ Parents of children with chronic conditions, who use multiple healthcare systems and providers, report portal underutilization and suggest increased use by both providers and patients may alleviate caregiver stress and the portal may serve as a potential modality for chronic care education and as a means to provide more effective patient/provider communication. ${ }^{9-12}$

In order for the portal to become a patient engagement tool, patients must first register and be willing to utilize the portal. ${ }^{7}$ This study will examine patient portal enrollment and activation patterns at a tertiary pediatric hospital in Southern California by race/ethnicity, preferred language, gender, and residential region. Given past findings within adult patient populations, it is hypothesized that racial/ethnic minorities will have low portal activation rates in a pediatric population. Findings of this study will help researchers and providers understand characteristics of portal use and disuse as well as identify factors to increase portal activation.

\section{Methods}

All procedures were approved by the appropriate administrative and university institutional review boards for the protection of human subjects. 


\section{Study Setting}

The study was conducted at a tertiary pediatric hospital and its affiliated network, which draws from 3 counties in Southern California, serving more than 750,000 children, ${ }^{13}$ and has approximately 4,900 unique outpatient visits per year. The pediatric hospital is located near an international border and its patients' racial/ethnic composition is approximately $45 \%$ Hispanic/Latino, 35\% White, and 10\% Asian. ${ }^{13}$ The study focused pediatric urology division patients. The pediatric urology division is the primary referral provider for the geographic area and manages care for children with chronic disease, resulting in a heterogeneous, robust patient sample in which to examine adoption patterns.

Patient medical care is tracked through the Epic EHR system (Verona, WI), which incorporates emergency department (ED), inpatient, outpatient (including satellite clinics), laboratory, and radiology input into an integrated system, sharing records within the organization. The Epic EHR has been fully operational at this location since 2010, while the portal called MyChart was first introduced in late 2010. An institutional-wide program incorporating pamphlets, targeted mailings (English/Spanish), flyers, and clinical staff directed patient education to encourage portal enrollment was conducted from 2011 to 2012.

\section{Patient Portal Activation}

MyChart's institutional configuration requires access to an internet connected computer and an up-to-date browser (such as Internet Explorer). MyChart includes the ability to securely email a physician's office, access test results, view a child's health summary, request prescription refills, request primary-care appointments, and view all scheduled appointments. Activation was a two- step process in which clinical staff provided an activation code to patient caregivers (or patients 12 or older) during their office visit, which allowed them to login and create a user name and password. Patients were also able to access MyChart through an iPhone or Android application, but were limited to viewing test results, upcoming appointments personal health summary, and messaging providers. Patients younger than 12 required a parent or legal guardian to activate an account. Pediatric patients age 12 or older, with parental permission, could their own accounts, though access to particular functions may have been limited by their parents. All of the enrollment materials as well as the MyChart portal were available in English and Spanish. Interpretation services were available for those needing assistance in other languages.

\section{Patients}

Activation of Epic's MyChart patient portal was retrospectively assessed from December 2010 to May 2016 for all patients with at least one visit with an outpatient urology visit using activation audit data. EHR data was examined from 10,464 patients aged 2 to 18 (calculated using date of birth and date of data extraction) who had at least one urology appointment and who met one of four portal activation categories: (1) activated (or caretaker activated) MyChart access code; (2) accepted but did not activate a code; (3) declined activation; or (4) activated and then deactivated their account. Demographic variables extracted included the patient's gender, preferred language, race, ethnicity, and zip code. Zip codes were used to assign patients within geographical regions. 


\section{Statistical Analysis}

Available median total household income and benefits census data by zip code ${ }^{14}$ were downloaded and matched with patient zip code. Summary statistics were calculated and associations among categorical demographic variables and the four portal status categories were analyzed using Pearson chi-square. Once bivariate relationships were identified, using a cutoff of a chi-square $p$-value of less than 0.05 as significant, an enter logistic regression model was used in which all significant variables were entered simultaneously to examine the interrelationship and the effect of identified variables on activation status (activated/not activated). The enter method is appropriate when there is a strong hypothesis about which variables belong in the model and produces an adjusted odds ratio, which takes into account the inter-relationship among many demographic variables. Activation was defined as having used a code and having a presently active account, while those who had refused, had not activated their code, or deactivated their code after activation were classified as not activated. All statistical analysis was conducted using SPSS version $24 .{ }^{15}$

\section{Results}

During the five and a half year period, 10,464 pediatric urology patients met criteria for analysis. Overall $46.5 \%$ of patients (or caregivers) who were offered an activation code, logged into MyChart (Table 1). Preferred language, ethnicity, and race of patients were significantly associated with portal adoption. Those who reported their preferred language as Spanish were significantly less likely to activate the portal $\left(\chi^{2}=895.9, p<.001\right)$ than English-speaking patients. Hispanic patients and those not reporting ethnicity $\left(\chi^{2}=412.02\right.$, $p<.001)$ were less likely than non-Hispanic patients to activate their portal. Those selfidentifying as Other (not White, Asian, or African American) $\left(\chi^{2}=437.7, p<.001\right)$ were less likely to activate their portal compared to Whites. Residential location was also associated with activation status $\left(\chi^{2}=240.3, p<.001\right)$. Those living in the central urban

region, which has some of the county's greatest language diversity lowest income areas were significantly less likely to activate their accounts. Median household income and benefits in 2014 inflation dollars by region are presented in Table 2 . Males were also less likely to activate MyChart $\left(\chi^{2}=10.2, p=.02\right)$. Of note, males represented $72 \%$ of the studied urology patients.

Language, gender, race, ethnicity, and residential region all demonstrated highly significant odds ratios in unadjusted bivariate logistics regression (Table 3), but in the saturated logistic regression model, controlling for all the variables in the model, language, race, and residential area remained significantly associated with activation status. Those reporting Spanish as their primary language were a third as likely to activate the portal (OR $0.33, p<$. 001) and those who reported a language other than English were 57\% as likely to report activation (OR 0.57, $p<.001$ ) as compared to primary English language patients. Compared to those who reported being White, individuals reporting their race as Other (OR 0.69. $p<$. 001) and those who identified as African American (OR 0.66, $p<.001$ ) were two-thirds as likely to activate; those who did not report a race (OR 0.36, $p<.001$ ) were a third as likely. Living in the suburbs was significant with two suburban areas, the North Central (OR 1.90, $p$ 
$<.001)$ and the Southern (OR 1.96, $p<.001)$ areas of the county twice as likely to activate portal accounts as those living in the urban Central region (Table 3).

\section{Discussion}

A retrospective analysis of pediatric urology patient portal activation within a large heterogeneous healthcare delivery system, demonstrated significantly different activation rates for patients by language preference, race, ethnicity, and geographic region. These differences suggest a continuing health care informatics divide among patients (and their caregivers), even with the increased focus on electronic enrollment as part of meaningful use mandates. The differences in activation rates indicates the need for further exploration of barriers and patient satisfaction with the portal. The results from this analysis identify patient groups who are not activating the portal and supports targeted patient education and recruitment.

Although the front office staff are bilingual and the portal invitation, activation directions, and electronic interface were available in Spanish, individuals who report Spanish as their primary language are not activating at similar rates to those who report English as their primary language. These pediatric findings are congruent with several adult studies and one pediatric study reporting individuals of color and those who report a language other than English as their primary language were less likely to obtain a portal account. ${ }^{3,8,9,16}$ Ketterer et al noted several sociodemographic disparities among portal adoption, including Hispanic ethnicity, which decreased portal activation odds approximately $25 \% .{ }^{17}$ Language was highly significant in this study. Controlling for other demographic factors, those reporting Spanish or another language other than English as the primary language were a third and half as likely to activate the portal, respectively. As a growing number of patients and families in the Southern California region identify Chinese and Arabic as their language preference, reducing potential linguistic issues as a barrier is critical for continued portal adoption.

More recently, Peacock et al suggest in addition to factors of race/ethnicity and language, individuals living in non-metropolitan areas are less likely to use the portal because they are not offered portal access as often as their urban counterparts. ${ }^{18}$ Different geographical adoption patterns were also noted in this study, with the most demographically diverse region the least likely to adopt the portal. While controlling for race, ethnicity, and language, this regional difference persisted, which may represent a variety of factors including limited internet access, receiving primary care at community clinics rather than within this healthcare system, and less general access to health care.

A significant study strength was the available statistical power for data analysis of demographic factors from a large heterogeneous healthcare delivery system that is the primary referral source for urologic conditions in the geographic area. This project demonstrated it is possible to summarize portal account activation and illustrated a notable and troubling disparity in activation. Such data regarding adoption patterns are critical if the portal is to become an active means of patient engagement with the clinical team and to serve as the patients' conduit to meaningful use of the EHR. Data such as these will add to 
the growing body of patient engagement and portal activation and will be able to advise clinicians and administrators in order to provide the desired patient-centered approaches for increased engagement, which recognize critical social factors and the need to overcome current barriers. For example, the urology division treats patients with a variety of chronic conditions, such as spina bifida, and the portal may be a way to engage vulnerable patient groups into the healthcare system.

In order to protect patient security, the portal activation process purposefully incorporates multiple steps, introducing several opportunities for a patient to become frustrated and to fail to complete. In recognition of the complexity of the activation process, a useful approach may be to provide more in-clinic opportunities to register as well as additional patient education about the utility of the portal during waiting times and as part of the engagement with clinical staff. In addition to understanding why accounts are or are not activated, results from this study also demonstrated many accounts are deactivated after enrollment. Understanding why the account is no longer used after the activation process has been completed is an area for further investigation.

There are several limitations to this retrospective analysis, including unavailable information about specific income levels (zip code and regional information were considered, but they do not provide individual level data.) as well as patient and caregiver health literacy levels, to evaluate the role of eHealth literacy and income as barriers to use. ${ }^{19}$ Caregivers, rather than patients were the probable portal user, are likely to have been female and have similar racial and ethnic backgrounds as the patient ${ }^{16,20}$, however their information is unknown. The patient's date of birth was available as part of the data download script parameters, but not available as a variable, so patterns of use in different age groups could not be evaluated. Without dates for non- activation, it was not possible to analyze adoption over time. Mobile phone and tablet use of MyChart is limited compared to the desktop usage, which may impact activation rates among those without regular computer access. Potential portal barriers include slow or non-existent internet access, data entry time, and data entry difficulty. ${ }^{21}$ Future studies should incorporate a measurement of patient/caregiver internet access. Given the current available data structure, the study did not look at other clinical factors that might have affected use such as diagnoses and condition severity. Because the healthcare system studied is the sole pediatric referral health care center for two large Southern California counties and part of a third, many of the patients may have received specialty care within this department but receive and are part of a primary care system elsewhere. This may have affected activation rates as many individuals did not view the study site as their home system.

\section{Conclusions}

A limited body of research, conducted primarily in academic settings among parents of children with chronic conditions, addresses the patient portal. This study conducted within the urology department of a large, integrated pediatric healthcare system serving a vast geographic area in Southern California demonstrates many patients have yet to activate their patient portal access and there are significant differences in adoption rates by primary language; race; and geographic location. Bilingual, bicultural educational materials, and 
electronic enrollment with clinical staff assistance may help facilitate patient portal adoption. This study identifies foci areas including health literacy, education level, overall literacy, household size, and family structure to include in further barrier assessment. The benefits of pediatric patient portal use to improve patient engagement, to improve transition to care, and to benefit research, can only be realized if individuals are enrolled in the portal.

\section{Acknowledgments}

This project was supported in part by grant number R00 HS022404 from the Agency for Healthcare Research and Quality. The content is solely the responsibility of the authors and does not necessarily represent the official views of the Agency for Healthcare Research and Quality.

\section{References}

1. What is a patient portal?. HealthIT.gov. https://www.healthit.gov/providers-professionals/faqs/whatpatient-portal. Updated 2 November, 2015. Accessed 11 March, 2017

2. Kruse CS, Argueta DA, Lopez L, et al. Patient and provider attitudes toward the use of patient portals for the management of chronic disease: a systematic review. J Med Internet Res. 2015; 17:e40. Internet. http://www.jmir.org/article/viewFile/jmir_v17i2e40/2. Accessed March 11, 2017. doi: 10.2196/jmir.3703 [PubMed: 25707035]

3. Griffin A, Skinner A, Thornhill J, et al. Patient portals: who uses them? What features do they use? And do they reduce hospital readmissions? Appl Clin Inform. 2016; 7:489. Internet. http:// www.ncbi.nlm.nih.gov/pubmed/25707035. Accessed March 11, 2017. doi: 10.4338/ACI-2016-01RA-0003 [PubMed: 27437056]

4. Emont, S. Measuring the impact of patient portals: what the literature tells us. California HealthCare Foundation Internet. http://www.chcf.org/publications/2011/05/measuring-impact-patient-portals. Accessed July11, 2014

5. Goldzweig CL, Orshansky G, Paige NM, et al. Electronic patient portals: evidence on health outcomes, satisfaction, efficiency, and attitudes: a systematic review. Ann Intern Med. 2013; 159:677. Internet. http://www.ncbi.nlm.nih.gov/pubmed/24247673. Accessed July 11, 2014. doi: 10.7326/0003-4819-159-10-201311190-00006 [PubMed: 24247673]

6. Rosen P, Spalding SJ, Hannon MJ, et al. Parent satisfaction with the electronic medical record in an academic pediatric rheumatology practice. J Med Internet Res. 2011; 13:e40. Internet. https:// www.jmir.org/2011/2/e40/. Accessed December 9, 2014. doi: 10.2196/jmir.1525 [PubMed: 21622292]

7. Irizarry T, DeVito Dabbs A, Curran CR. Patient portals and patient engagement: a state of the science review. J Med Internet Res. 2015; 17:e148.doi: 10.2196/jmir.4255 [PubMed: 26104044]

8. Goel MS, Brown TL, Williams A, et al. Disparities in enrollment and use of an electronic patient portal. J Gen Intern Med. 2011; 26:1112.doi: 10.1007/s11606-011-1728-3 [PubMed: 21538166]

9. Byczkowski TL, Munafo JK, Britto MT. Variation in use of internet-based patient portals by parents of children with chronic disease. Arch Pediatr Adolesc Med. 2011; 165:405. Internet. http:// jamanetwork.com/journals/jamapediatrics/fullarticle/384633. Accessed December 10, 2014. doi: 10.1001/archpediatrics.2011.55 [PubMed: 21536954]

10. Britto MT, Hesse EA, Kamdar OJ, et al. Parents' perceptions of a patient portal for managing their child's chronic illness. J Pediatr. 2013; 163:280. Internet. http://www.ncbi.nlm.nih.gov/pubmed/ 23541773. Accessed 2 August, 2014. doi: 10.1016/j.jpeds.2013.02.041 [PubMed: 23541773]

11. Bush RA, Stahmer AC, Connelly CD. Exploring perceptions and use of the electronic health record by parents of children with autism spectrum disorder: a qualitative study. Heal Informatics J. 2016; 22:702.doi: $10.1177 / 1460458215581911$

12. How to optimize patient portals for patient engagement. HealthIT.gov. https://www.healthit.gov/ sites/default/files/nlc_how_to_optimizepatientportals_for_patientengagement.pdf. Published May, 2013. Accessed 11 March, 2017 
13. All data: San Diego County. Kidsdata.org. http://www.kidsdata.org/region/368/san-diego-county/ results. Accessed 11 March, 2017

14. San Diego's Regional Planning Agency (SANDAG). http://datasurfer.sandag.org/. http:// www.sandag.org/uploads/publicationid/publicationid_2001_20213.pdf. Updated January, 2016. Accessed 15 March, 2017

15. IBM Corp. Released 2016. IBM SPSS Statistics for Windows, Version 24.0. Armonk, NY: IBM Corp;

16. Weingart SN, Rind D, Tofias Z, et al. Who uses the patient internet portal? the patient site experience. J Am Med Inform Assoc. 2006; 13:91. Internet. https://www.ncbi.nlm.nih.gov/pmc/ articles/PMC1380201/. Accessed 1 January, 2006. doi: 10.1197/jamia.M1833 [PubMed: 16221943]

17. Ketterer T, West DW, Sanders VP, et al. Correlates of patient portal enrollment and activation in primary care pediatrics. Acad Pediatr. 2013; 13:264. Internet. http://www.ncbi.nlm.nih.gov/ pubmed/23680344. Accessed 2 August, 2014. doi: 10.1016/j.acap.2013.02.002 [PubMed: 23680344]

18. Peacock, S., Reddy, A., Leveille, SG., et al. Patient portals and personal health information online: perception, access, and use by US adults [published online jul 12, 2016]. J Am Med Inform Assoc. Internet. https://academic.oup.com/jamia/article-lookup/doi/10.1093/jamia/ocw095. Accessed 11 March, 2017

19. Noblin AM, Wan TTH, Fottler M. The impact of health literacy on a patient's decision to adopt a personal health record. Perspect Health Inf Manag. 2012; 9:1e. Internet https:// www.ncbi.nlm.nih.gov/pmc/articles/PMC3510648/. Accessed 11 March 2017.

20. Palen TE. Association of online patient access to clinicians and medical records with use of clinical services. JAMA. 2012; 308:2012. Internet. http://jamanetwork.com/journals/jama/fullarticle/ 1392562. Accessed 11 March, 2017. doi: 10.1001/jama.2012.14126 [PubMed: 23168824]

21. Urowitz S, Wiljer D, Dupak K, et al. Improving diabetes management with a patient portal: a qualitative study of diabetes self-management portal. J Med Internet Res. 2012; 14:e158. Internet. https://www.ncbi.nlm.nih.gov/pmc/articles/PMC3510725/. Accessed 11 March, 2017. doi: 10.2196/jmir.2265 [PubMed: 23195925] 


\section{을 \\ 골}

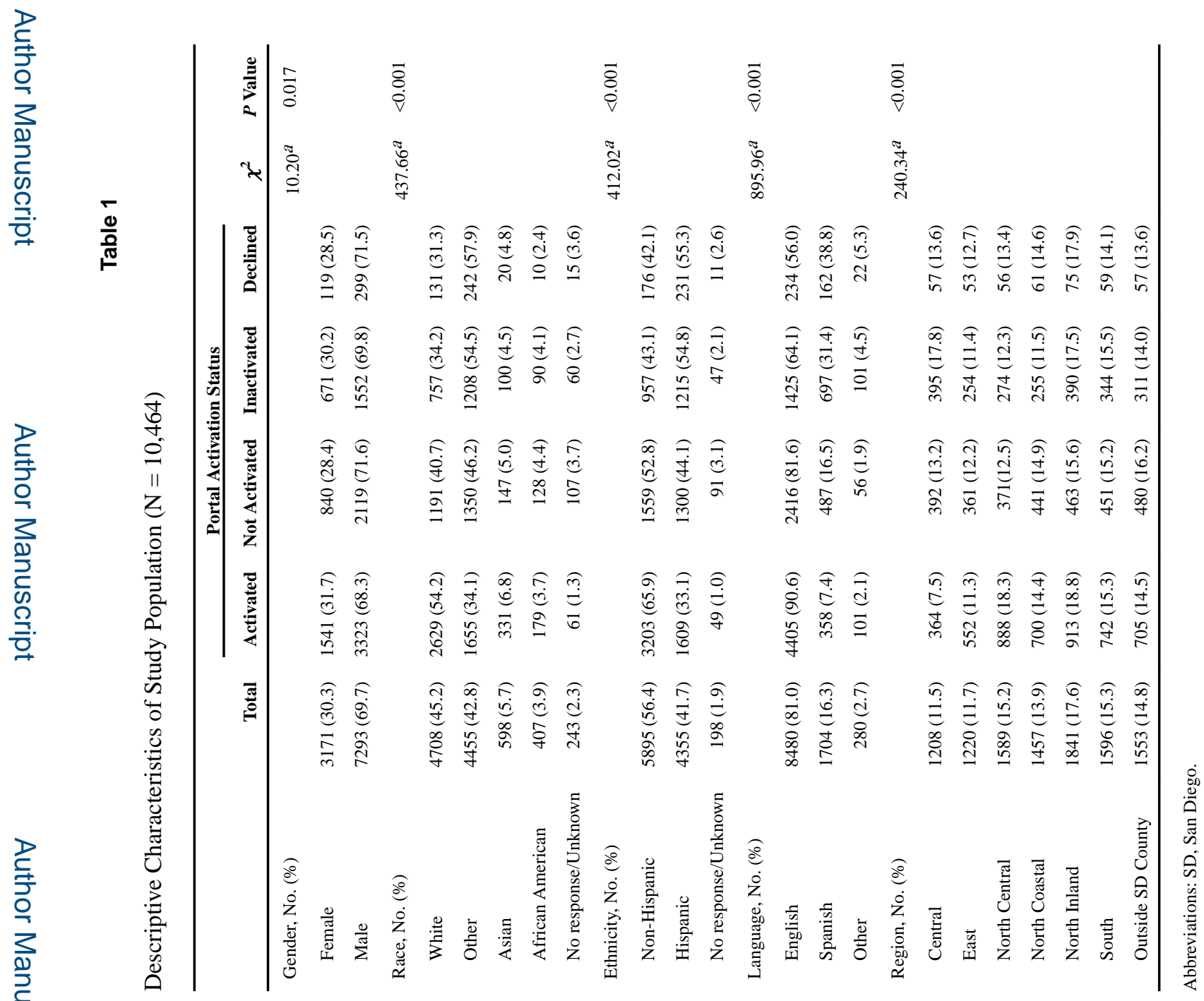


Table 2

Estimated Median Household Income and Benefits in 2014 Inflation-Adjusted Dollars ${ }^{a}$

\begin{tabular}{llcc}
\hline Region & No. of Patients (\%) & Mean \pm SD & 95\% CI \\
\hline Central $^{b}$ & $1208(14)$ & $44,403.0 \pm 11,030.1$ & $43,780.4-45,025.6$ \\
East $^{c}$ & $1220(14)$ & $61,267.0 \pm 13,158.2$ & $60,527.9-62,006.1$ \\
North Central $^{d}$ & $1594(18)$ & $87,412.0 \pm 25,297.4$ & $86,169.2-88,654.8$ \\
North Coastal $^{f}$ & $1459(16)$ & $74,795.7 \pm 23,441.4$ & $73,591.9-75,999.5$ \\
North Inland & $1842(21)$ & $76,577.2 \pm 26,895.3$ & $75,348.2-77,806.2$ \\
South & $1596(18)$ & $59,749.4 \pm 20,482.0$ & $58,743.8-60,755.0$ \\
Total & 8919 & $68,759.0 \pm 25,533.9$ & $68,229.0-69,289.0$ \\
\hline
\end{tabular}

Abbreviations: SD, standard deviation; CI, confidence interval of the mean.

${ }^{a}$ ANOVA: $\mathrm{F}=697.37, p<.001$ using Tukey Post-Hoc tests.

${ }^{b}$ Central Region significantly different from all other regions

${ }^{c}$ East Region significantly different from all other regions except South

${ }^{d}$ North Central Region significantly different from all other regions

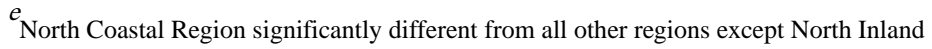




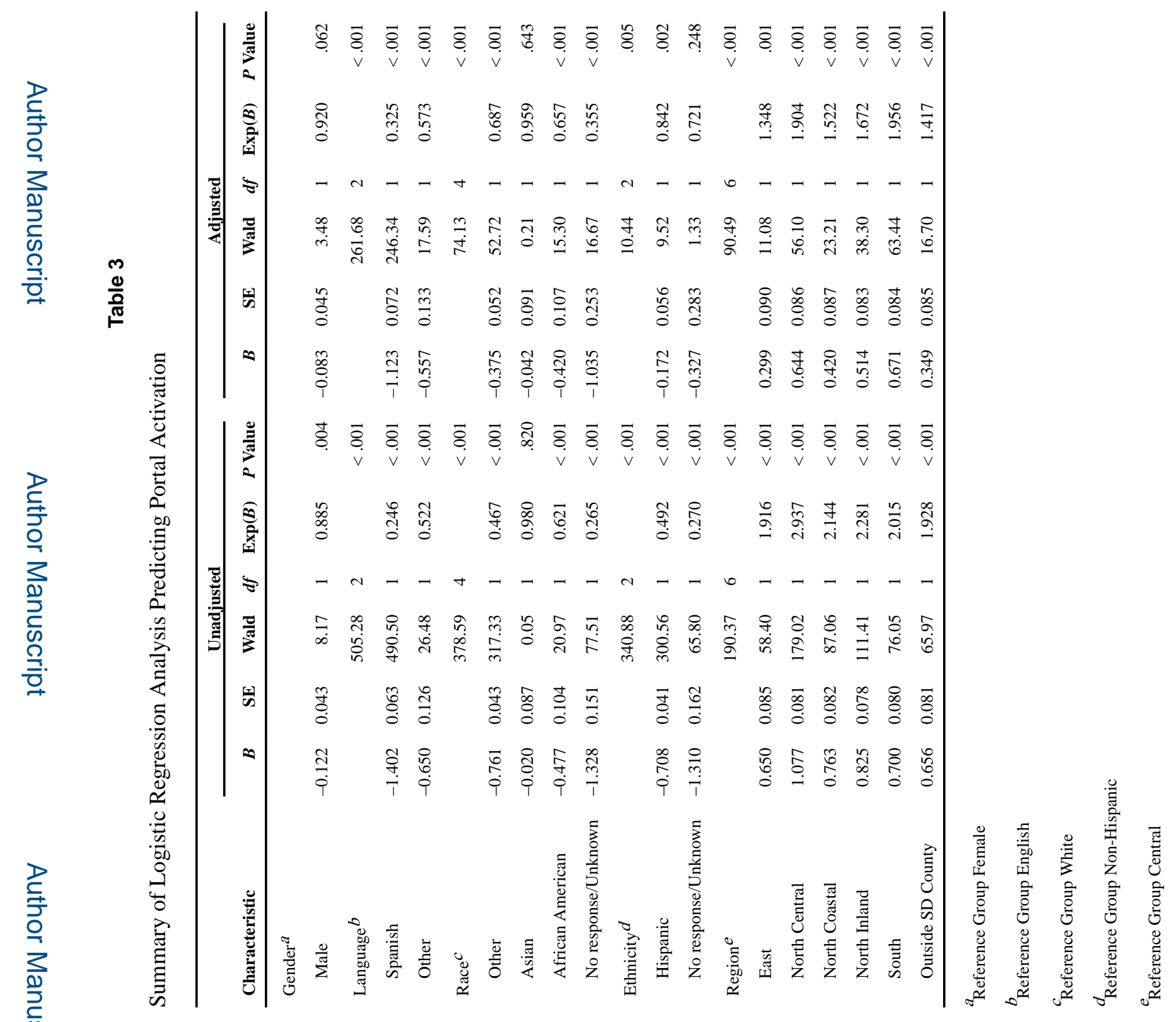

